\title{
Thermally stable bipolar host materials for high efficiency phosphorescent green and
}

\section{blue organic light-emitting diodes}

Qingchen Dong, ${ }^{\text {a, }}$ Feifei Tai, ${ }^{a}$ Hong Lian, ${ }^{a}$ Zheng Chen, ${ }^{a}$ Mingming Hu, ${ }^{b}$ Jinhai Huang, ${ }^{\text {b, }}$ and Wai-Yeung Wong $^{\mathrm{a}^{*}}$

${ }^{a}$ MOE Key Laboratory of Interface Science and Engineering in Advanced Materials and Research Center of Advanced Materials Science and Technology, Taiyuan University of Technology, 79 Yingze West Street, Taiyuan 030024, P. R. China. Fax: +86-351-6010311; Tel.: +86-351-6010311; E-mail:dongqingchen@tyut.edu.cn.

${ }^{b}$ Shanghai Taoe Chemical Technology Co., Ltd, Shanghai, P. R. China. Fax: +86-21-64252758; Tel.: +86-21-64252758; E-mail: dele12@163.com 


\section{Abstract}

Two bipolar host materials H1 ( $N$-linked carbazole) and $\mathbf{H 2}$ ( $C$-3-linked carbazole) via changing the substitution position of triazine and carbazole moieties are reported. Their structural and physical properties were fully characterized. The thermal analysis demonstrated that both H1 and $\mathbf{H 2}$ are of high thermal stability. The triplet energy levels of $\mathbf{H 1}$ and $\mathbf{H 2}$ were determined to be 2.64 and $2.7 \mathrm{eV}$, respectively. Both green and blue OLEDs with $\mathbf{H 1}$ and $\mathbf{H 2}$ acting as host materials were fabricated to investigate their performances in PHOLED. The green PHOLED based on $\mathbf{H 1}$ exhibits the best performance with the maximum current efficiency, maximum power efficiency and maximum brightness of $51.7 \mathrm{~cd} / \mathrm{A}, 37.8 \mathrm{~lm} / \mathrm{W}$ and $132800 \mathrm{~cd} / \mathrm{m}^{2}$, respectively, while the blue PHOLED based on $\mathbf{H 2}$ shows the best performance with the maximum current efficiency, maximum power efficiency and maximum brightnes s of $21.6 \mathrm{~cd} / \mathrm{A}$, $17.2 \mathrm{~lm} / \mathrm{W}$ and $16640 \mathrm{~cd} / \mathrm{m}^{2}$, respectively. The characteristic measurements of OLED devices reveal that both $\mathbf{H 1}$ and $\mathbf{H 2}$ are promising host materials for practical application. By comparing the commercial host materials of 4,4'-bis(carbazol-9-yl)biphenyl (CBP) and N,N-dicarbazoyl3,5-benzene $(\mathrm{mCP})$, the power efficiencies of green and blue PHOLED were boosted by $22.3 \%$ and $75.5 \%$, respectively. 


\section{Graphical abstract}
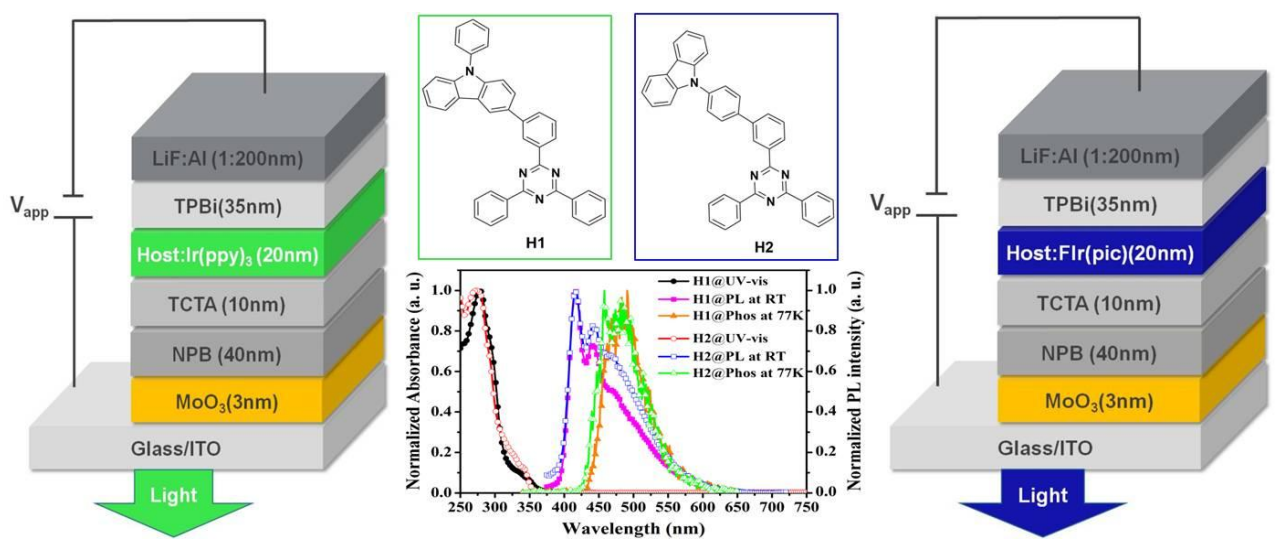


\section{Keywords}

Triazine derivatives; Carbazole derivatives; Bipolar host materials; Phosphorescent organic light-emitting diodes. 


\section{Introduction}

Phosphorescent organic light-emitting diodes (PHOLEDs) have attracted huge attention from academic to industry in recent years due to their appealing commercial interest in the field of flat panel display, solid-sate lighting and virtual reality [1-4]. Due to the challenges of materials and life-time faced in the PHOLED industry, numerous works have been done to explore novel materials including the carrier transport materials, emitting materials and host materials, etc. to push the development of PHOLEDs [5-10]. The phosphorescent host materials are crucial which serve as the recombination center for electrons and holes to generate the electronically excited states [11-14]. Generally, an appropriate host material should meet the following intrinsic requirements: firstly, a triplet energy gap $\left(\mathrm{E}_{\mathrm{T}}\right)$ larger than that of the dopant to prevent reverse energy transfer from the guest back to the host; secondly, good carrier transporting properties to balance the charge flux and confine the triplet exciton within the emitting layer and reduce the driving voltage; thirdly, good thermal and morphological stability to prolong the operation lifetime of the OLED $[15,16]$. Among the host materials, bipolar molecules, which consist of an electron-donating moiety and an electron-withdrawing moiety, usually exhibit excellent performance in PHOLEDs. This is because the bipolar molecules have admirable carrier transport ability for both electron and hole, which thus could balance the carrier mobility. Limitation of $\pi-$ conjugation with silicon or $\mathrm{sp}^{3}$ carbon or increase of distortion of the molecular structure could enhance the triplet energy level of bipolar host materials [17-21]. Nowadays, various organic functional groups have been investigated to explore suitable bipolar host materials. The carbazole derivatives exhibit suitable energy gap and high fluorescent quantum yield, [22-26] and the triazine derivatives are good electron-transport materials due to their electron deficiency $[27,28]$. In 2015, Lee et al. reported one host material TrzmPCz based on triazine and carbazole for blue 
PHOLED, and the current efficiency and power efficiency are $32 \mathrm{~cd} / \mathrm{A}$ and $21.5 \mathrm{~lm} / \mathrm{W}$, respectively. ${ }^{29}$ Lee and co-workers attributed the excellent performance of TrzmPCz to the distorted molecular structure through theoretical calculation comparison of $\mathrm{TrzmPCz}$ and the control compound TrzPCz. However, the authors did not conduct the synthesis of control compound TrzPCz, and the practical physical properties and OLED fabrication based on TrzPCz were also not performed.

Hence, in this study, we designed and synthesized two bipolar host materials $\mathbf{H 1}$ and $\mathbf{H 2}$ through different linkage mode of triazine and carbazole moieties. The molecular structure of $\mathbf{H 1}$ is equal to the TrzPCz as mentioned in the previous report by Lee et al. Both green and blue phosphorescent OLEDs based on $\mathbf{H 1}$ and $\mathbf{H 2}$ were fabricated. H1 exhibited the best performance with the maximum current efficiency and power efficiency of $51.5 \mathrm{~cd} / \mathrm{A}$ and $37.8 \mathrm{~lm} / \mathrm{W}$ for green PHOLED, while $\mathbf{H 2}$ displayed the highest current efficiency and power efficiency of $21.6 \mathrm{~cd} / \mathrm{A}$ and $17.2 \mathrm{~lm} / \mathrm{W}$ for blue PHOLED, respectively. By comparing with the commercial host materials of 4,4'-bis(carbazol-9-yl)biphenyl (CBP) and N,N-dicarbazoyl-3,5-benzene (mCP), the power efficiency of green and blue PHOLED was enhanced by $22.3 \%$ and $75.5 \%$, respectively.

\section{Experimental}

\subsection{General information}

All the solvents and chemicals that were used in the synthetic route were of reagent grades and purchased from J \& K Chemical Co. and Aladdin Chemical Co. without further purification. The starting compounds 1, 2 and 3 were purchased from Shanghai Taoe Chemical Technology Co., Ltd for synthesis of target products directly. Tetrahydrofuran (THF) was purified by distillation over sodium under a $\mathrm{N}_{2}$ atmosphere prior to use. All reactions and manipulations were carried out under a $\mathrm{N}_{2}$ atmosphere. All column chromatography was performed on silica gel (300 - 400 mesh) 
as the stationary phase in the column. All materials were purified further by vacuum sublimation prior to fabrication of OLED devices.

The ${ }^{1} \mathrm{H}$ and ${ }^{13} \mathrm{C}$ NMR spectra were recorded on a Bruker AM 400 spectrometer with tetramethylsilane as the internal standard. High-resolution mass spectra were measured on a Waters LCT Premier XE spectrometer. The ultraviolet-visible (UV-Vis) absorption spectra were obtained on a Varian Cary 500 spectrophotometer. Photoluminescence (PL) spectra were recorded on a Varian-Cary fluorescence spectrophotometer. The cyclic voltammetry experiments were performed using a Versastat II electrochemical work station (Princeton applied research) using a conventional three-electrode configuration with a glassy carbon working electrode, a Pt wire counter electrode, and a regular calomel reference electrode in saturated $\mathrm{KCl}$ solution. The oxidation and reduction potentials were measured in dichloromethane/acetonitrile (7:3, v/v) solution containing of $0.1 \mathrm{M}$ tetra- $n$-butylammonium perchlorate (TBAP) as the supporting electrolyte at a scan rate of $100 \mathrm{mV} / \mathrm{s}$. The differential scanning calorimetry (DSC) analysis was performed on a DSC Q2000 V24.11 Build 124 instrument with a heating scan rate of $10{ }^{\circ} \mathrm{C} / \mathrm{min}$ from $0{ }^{\circ} \mathrm{C}$ to $250{ }^{\circ} \mathrm{C}$ under nitrogen atmosphere. Thermogravimetric analysis (TGA) was carried out on the TGA instrument by measuring weight loss of samples with a heating scan rate of $10^{\circ} \mathrm{C}$ /min from $50{ }^{\circ} \mathrm{C}$ to $800{ }^{\circ} \mathrm{C}$ under nitrogen.

\subsection{Preparation of 3-(3-(4,6-diphenyl-1,3,5-triazin-2-yl)phenyl)-9-phenyl-9H-carbazole (H1)}

\section{and 9-(3'-(4,6-diphenyl-1,3,5-triazin-2-yl)-[1,1'-biphenyl]-4-yl)-9H-carbazole (H2)}

In a $100 \mathrm{~mL}$ one-neck flask, (9-phenyl-9H-carbazol-3-yl)boronic acid (1) (0.50 g, $1.74 \mathrm{mmol})$ and compound 2 (0.56 g, $1.44 \mathrm{mmol})$ were dissolved in solution of THF $(20 \mathrm{~mL})$ and $2 \mathrm{M} \mathrm{K}_{2} \mathrm{CO}_{3}(20 \mathrm{~mL})$, followed by addition of $\mathrm{Pd}(\mathrm{OAc})_{2}(16.1 \mathrm{mg}, 0.072 \mathrm{mmol})$ and $\mathrm{X}$-phos $(50.7 \mathrm{mg}, 0.144 \mathrm{mmol})$ under a nitrogen atmosphere. The mixture was heated to reflux for $6 \mathrm{~h}$. The completion of the reaction was verified by spot TLC. The resulting mixture was extracted by dichloromethane for 3 times, then the 
organic phase was collected and concentrated by a rotary evaporator. The crude material was purified by silica gel column chromatography using a petroleum and dichloromethane mixture (v:v $=6: 1)$ as the eluent to obtain the desired product (H1) as a white solid $(0.52 \mathrm{~g}, 66 \%) .{ }^{1} \mathrm{H} \mathrm{NMR}\left(\mathrm{CDCl}_{3}, 400 \mathrm{MHz}\right.$,

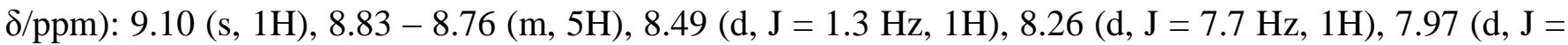
$7.8 \mathrm{~Hz}, 1 \mathrm{H}), 7.81(\mathrm{dd}, \mathrm{J}=8.5,1.7 \mathrm{~Hz}, 1 \mathrm{H}), 7.72-7.55(\mathrm{~m}, 13 \mathrm{H}), 7.46(\mathrm{~d}, \mathrm{~J}=3.7 \mathrm{~Hz}, 2 \mathrm{H}), 7.36-7.32(\mathrm{~m}$, $1 \mathrm{H}) ;{ }^{13} \mathrm{C}$ NMR $\left(\mathrm{CDCl}_{3}, 101 \mathrm{MHz}, \delta / \mathrm{ppm}\right): 171.75,137.65,136.79,136.28,133.16,132.55,131.58$, $129.96,129.09,128.69,127.84,127.62,127.19,126.26(\mathrm{~s}), 125.71,123.97,123.47,120.52,120.17$, 119.13, 110.09; HRMS (ESI, $m / z)$ : $[\mathrm{M}+\mathrm{H}]^{+}$calcd for $\mathrm{C}_{39} \mathrm{H}_{26} \mathrm{~N}_{4}, 550.2200$, found 551.2239; IR (KBr): $1366\left(v_{\mathrm{C}-\mathrm{N}}\right) \mathrm{cm}^{-1}, 1595,1528,1498,1450\left(v_{\mathrm{C}=\mathrm{C}}\right) \mathrm{cm}^{-1}, 1626\left(v_{\mathrm{C}=\mathrm{N}}\right) \mathrm{cm}^{-1}, 3056\left(v_{\mathrm{C}=\mathrm{C}-\mathrm{H}}\right) \mathrm{cm}^{-1} ; \mathrm{T}_{\text {decomp }}\left({ }^{\circ} \mathrm{C}\right)$ : $402.5^{\circ} \mathrm{C}$ for $\mathbf{H 1}$.

The synthetic procedure of $\mathbf{H 2}$ is similar to that of $\mathbf{H 1}$, in which (4-(9H-carbazol-9yl)phenyl)boronic acid (3) was used to replace compound 1. The final product (H2) was obtained as a white solid $(0.57 \mathrm{~g}, 72 \%) .{ }^{1} \mathrm{H}$ NMR $\left(\mathrm{CDCl}_{3}, 400 \mathrm{MHz}, \delta / \mathrm{ppm}\right): 9.10(\mathrm{~s}, 1 \mathrm{H}), 8.83-8.76(\mathrm{~m}$, 5H), $8.49(\mathrm{~d}, \mathrm{~J}=1.3 \mathrm{~Hz}, 1 \mathrm{H}), 8.26(\mathrm{~d}, \mathrm{~J}=7.7 \mathrm{~Hz}, 1 \mathrm{H}), 7.97(\mathrm{~d}, \mathrm{~J}=7.8 \mathrm{~Hz}, 1 \mathrm{H}), 7.81(\mathrm{dd}, \mathrm{J}=8.5$, $1.7 \mathrm{~Hz}, 1 \mathrm{H}), 7.72-7.55(\mathrm{~m}, 13 \mathrm{H}), 7.46(\mathrm{~d}, \mathrm{~J}=3.7 \mathrm{~Hz}, 2 \mathrm{H}), 7.36-7.32(\mathrm{~m}, 1 \mathrm{H}) ;{ }^{13} \mathrm{C}$ NMR $\left(\mathrm{CDCl}_{3}, 101 \mathrm{MHz}, \delta / \mathrm{ppm}\right): 171.85,140.80,136.16,132.56,129.07,128.71,127.45-127.25$, 126.03, 123.61, 120.42, $119.99,109.87$; HRMS (ESI, m/z): $[\mathrm{M}+\mathrm{H}]^{+}$calcd for $\mathrm{C}_{39} \mathrm{H}_{26} \mathrm{~N}_{4}$, 550.2200, found 551.2239; IR (KBr): $1357\left(v_{\mathrm{C}-\mathrm{N}}\right) \mathrm{cm}^{-1}, 1596,1520,1482,1450\left(v_{\mathrm{C}=\mathrm{C}}\right) \mathrm{cm}^{-1}, 1628$ $\left(v_{\mathrm{C}=\mathrm{N}}\right) \mathrm{cm}^{-1}, 3056\left(v_{\mathrm{C}=\mathrm{C}-\mathrm{H}}\right) \mathrm{cm}^{-1} ; \mathrm{T}_{\mathrm{decomp}}\left({ }^{\circ} \mathrm{C}\right): 416.8^{\circ} \mathrm{C}$ for $\mathbf{H 2}$.

\subsection{Fabrication and measurement of OLEDs}

The OLED devices were fabricated through vacuum thermal evaporation technology according to the method modified from our previous approach [30]. Device with an area of $3 \mathrm{~mm} \times 3 \mathrm{~mm}$ was fabricated by vacuum deposition (at $1 \times 10^{-6}$ Torr) of functional organic layers on the indium-tin-oxide (ITO)/glass 
substrate which has a sheet resistance of $25 \Omega /$ square. The ITO/glass substrate was cleaned sequentially by detergent, de-ionized water, acetone, ethanol and then dried in the oven at $120{ }^{\circ} \mathrm{C}$. Afterwards, the clean ITO glass substrate was treated with oxygen plasma for $8 \mathrm{~min}$.

All the other organic layers were deposited at a rate of $1.0 \AA / \mathrm{s}$ sequentially. The cathode was completed through thermal deposition of $\operatorname{LiF}(1 \mathrm{~nm})$ at a deposition rate of $0.1 \AA / \mathrm{s}$, then $\mathrm{Al}$ metal $(200 \mathrm{~nm})$ was deposited through thermal evaporation at a rate of $5.0 \AA$ As. The EL spectra were measured by a Spectrascan PR655 photometer. The current-voltage-luminance characteristics (I-V-L) were measured by a computer-controlled Keithley 2400 sourcemeter integrated with a TOPCOM BM-7 luminance meter. Current efficiency and power efficiency were calculated from the plot of I-V-L. All samples were characterized immediately after thin films deposition without encapsulation at room temperature. AFM was measured to investigate the surface morphology of the thin films of CBP, $\mathbf{m C P}, \mathbf{H 1}$ and $\mathbf{H 2}$.

\section{Results and discussion}

\subsection{Synthesis and characterization}

Chemical structures and the detailed synthetic protocols of target compounds $\mathbf{H 1}$ and $\mathbf{H 2}$ are summarized in Scheme 1. Synthesis of $\mathbf{H 1}$ and $\mathbf{H 2}$ is very simple and can be achieved by one step: (9-phenyl-9H-carbazol-3-yl)boronic acid (1) and (4-(9H-carbazol-9-yl)phenyl)boronic acid (3) were allowed to react with 2-(3-bromophenyl)-4,6-diphenyl-1,3,5-triazine, respectively, through Suzuki coupling to give rise to the target compounds in good yield. Flash column chromatography was performed to purify the final products. All the compounds were well characterized by ${ }^{1} \mathrm{H}$ NMR, ${ }^{13} \mathrm{C}$ NMR as well as the high-resolution mass spectrometry (HRMS).

\subsection{Thermal properties}

The thermal properties of compounds $\mathbf{H 1}$ and $\mathbf{H 2}$ were characterized by thermal gravimetric analyses (TGA) and differential scanning calorimetry (DSC) under a nitrogen stream. As shown 
in Fig. 1 and Table 1, the TGA data reveal that the onset decomposition temperatures $\left(\mathrm{T}_{\mathrm{d}}\right.$, corresponding to $5 \%$ weight loss) of these three materials are close to $400{ }^{\circ} \mathrm{C}$ implying that they are thermally stable. By analysis of the DSC traces, we found that, the endothermic glass transition temperatures $\left(\mathrm{T}_{\mathrm{g}}\right)$ for $\mathrm{H1}$ and $\mathbf{H 2}$ are determined to be 95 and $100{ }^{\circ} \mathrm{C}$ which is desirable for high-performance OLEDs. The high thermal stability of the new host materials is crucial for enhancing the film morphology and device lifetime by reducing the crystallization and phase separation during device operation.

\subsection{Photophysical properties}

To investigate the photophysical properties of compounds $\mathbf{H 1}$ and $\mathbf{H 2}$, their UV-Vis absorption spectra, photoluminescence (PL) spectra (at room temperature in THF) and phosphorescence spectra (at 77K in 2-methyl THF) were measured and presented in Fig. 2. The detailed data are listed in Table 1. As shown in Fig. 1, both $\mathbf{H 1}$ and $\mathbf{H 2}$ exhibit one major absorption bands at around $280 \mathrm{~nm}$ in their UV-Vis absorption spectra, which can be derived from the $\pi \rightarrow \pi^{*}$ transitions. The secondary, weak absorption peaks that are located at around $340 \mathrm{~nm}$ can be interpreted as arising from the CT states of the compounds [31]. The optical energy band gaps $\left(\mathrm{E}_{\mathrm{g}}\right)$ of $\mathrm{H1}$ and $\mathbf{H 2}$ that can be obtained from the threshold of the absorption spectra in THF are calculated to be $3.78 \mathrm{eV}$, and $3.62 \mathrm{eV}$, respectively. From the PL spectra of $\mathbf{H 1}$ and $\mathbf{H 2}$, we can see that, there are two emission maxima located at $416 \mathrm{~nm}$ and $441 \mathrm{~nm}$. From the phosphorescence spectra in the frozen 2-methyl THF at $77 \mathrm{~K}$, the triplet energies $\left(\mathrm{E}_{\mathrm{T}}\right)$ of $\mathbf{H 1}$ and $\mathbf{H 2}$ are determined to be $2.64 \mathrm{eV}$ and $2.70 \mathrm{eV}$, respectively, which were calculated on the basis of the short wavelength peaks. Moreover, we also investigated the quantum yield and lifetime of these two compounds, the results of which were listed in Table 1 . These photophysical properties of $\mathbf{H 1}$ and $\mathbf{H 2}$ allow them suitable in fabricating green and blue PHOLEDs [32].

\subsection{Electrochemical properties and energy levels}


The electrochemical properties of $\mathbf{H 1}$ and $\mathbf{H} 2$ were investigated by cyclic voltammetry (CV) and the first oxidation potentials were used to determine the highest occupied molecular orbital (HOMO) and lowest unoccupied molecular orbital (LUMO) energy levels (Fig. 3). The CV curves were measured in acetonitrile (0.1 M TBAP as the supporting electrolyte; scan rate: 100 $\mathrm{mV} \mathrm{s}^{-1}$ ), with SCE reference electrode (ferrocene/ferrocenium $\left(\mathrm{Fc}_{\mathrm{Fc}} \mathrm{Fc}^{+}\right.$) redox couple as the external standard), a glassy-carbon working electrode and a platinum-wire counter electrode. The onset oxidation potentials of both $\mathbf{H 1}$ and $\mathbf{H 2}$ were measured to be $1.29 \mathrm{eV}$ from the cyclic voltammograms. The HOMO values for $\mathbf{H 1}$ and $\mathbf{H 2}$ were calculated to be $-6.09 \mathrm{eV}$. According to

the formula $\mathrm{E}_{\mathrm{LUMO}}=\mathrm{E}_{\mathrm{HOMO}}+\mathrm{E}_{\mathrm{g}}$, the corresponding LUMO values of $\mathbf{H 1}$ and $\mathbf{H} \mathbf{2}$ were determined to be -2.87 and $-2.70 \mathrm{eV}$. These data can guarantee the outstanding carriers injection ability of $\mathbf{H 1}$ and $\mathbf{H} \mathbf{2}$ in green and blue phosphorescent OLEDs [33,34].

\subsection{Carrier transport properties}

Bipolar charge transport property of host material is one of the most important factors to contribute to the high performance of PhOLEDs. To evaluate the charge-transporting character of $\mathbf{H 1}$ and $\mathbf{H 2}$, hole-only devices with configuration of $\mathrm{ITO} / \mathrm{MoO}_{3}(3 \mathrm{~nm}) / \mathrm{NPB}(10 \mathrm{~nm}) / \mathrm{host}$ (40 $\mathrm{nm}) / \mathrm{NPB}(10 \mathrm{~nm}) / \mathrm{Al}(200 \mathrm{~nm})$ and electron-only devices with configuration of ITO/TPBi (10 $\mathrm{nm}) /$ host $(40 \mathrm{~nm}) / \mathrm{TPBi}(10 \mathrm{~nm}) / \mathrm{LiF}(1 \mathrm{~nm}) / \mathrm{Al}(200 \mathrm{~nm})$ were fabricated, respectively. In holeonly devices, the N,N'-diphenyl-N,N'-bis(1,1'-biphenyl)-4,4'-diamine (NPB)/Al interface was designed to prevent electron injection due to the large electron barrier of $1.8 \mathrm{eV}$. In the electrononly devices, a thin layer of 2,2',2' '-(1,3,5-benzinetriyl)-tris(1-phenyl-1-H-benzimidazole) (TPBi) was inserted between the ITO anode and the host layer to prevent hole injection due to the deep HOMO level $(-6.2 \mathrm{eV})$ of TPBi and thus the large hole barrier $(1.5 \mathrm{eV})$. As shown in Fig. 4, both hole-only and electron-only devices of $\mathbf{H 1}$ and $\mathbf{H 2}$ exhibit significantly high current densities in the voltage range typically suitable for OLEDs, indicating the bipolar charge-transport feature of 
them. Moreover, we can learn that both $\mathbf{H 1}$ and $\mathbf{H 2}$ are good at electron transportation by comparing with $\mathrm{CBP}$ and $\mathrm{mCP}$. It should be noted that the hole-transporting ability of $\mathbf{H 1}$ is better than H2, which may be attributed to the more coplanar conformation of $\mathbf{H 1}$ due to the steric effect. As reported previously, the more coplanar conformation is favored in the forming of high quality film, which thus benefits charge hopping, transportation and higher current densities in devices [35].

\subsection{Phosphorescent OLEDs}

To investigate the electroluminescent performances of $\mathbf{H 1}$ and $\mathbf{H 2}$ serving as the host materials, green and blue PHOLED devices based on $\mathbf{H 1}$ and $\mathbf{H 2}$ were fabricated. The device structures (Fig. 5a and 5c) were as follows: ITO/ $\mathrm{MoO}_{3}(3 \mathrm{~nm}) / \mathrm{NPB}(40 \mathrm{~nm}) / \mathrm{TCTA}(10 \mathrm{~nm}) / \mathrm{Host}: \operatorname{Ir}(\mathrm{ppy})_{3}(20$ nm, $8 \mathrm{wt} \%) / \mathrm{TPBi}(35 \mathrm{~nm}) / \mathrm{LiF}(1 \mathrm{~nm}) / \mathrm{Al}(200 \mathrm{~nm})$ for green OLEDs (denoted as H1-G and H2G, respectively) and $\mathrm{ITO} / \mathrm{MoO}_{3}(3 \mathrm{~nm}) / \mathrm{NPB}(40 \mathrm{~nm}) / \mathrm{TCTA}(10 \mathrm{~nm}) / \mathrm{Host}:$ FIrpic $(20 \mathrm{~nm}, 8$ wt\%)/TPBi $(35 \mathrm{~nm}) / \mathrm{LiF}(1 \mathrm{~nm}) / \mathrm{Al}(200 \mathrm{~nm})$ for blue OLEDs (denoted as H1-B and H2-B, respectively). $\mathrm{MoO}_{3}, \mathrm{NPB}$ and 4,4,4-tris(N-carbazolyl)triphenylamine (TCTA) served as the hole injection layer (HIL) and hole transport layers (HTL), respectively, the emitting layer (EML) was composed of $8 \mathrm{wt} \% \operatorname{Ir}(\mathrm{ppy})_{3}$ or bis[(4,6-difluorophenyl)-pyridinato-N,C2']c(picolinate)iridium(III) (Firpic) doped into host materials, TPBi acted as the electron transport layer (ETL) and LiF was employed as the electron injection layer (EIL). For comparison, the control devices of green OLED and blue OLED with CBP and $\mathrm{mCP}$ as host materials were also fabricated, denoted as Control-G and Control-B, respectively. Fig. 5b and 5d display the relevant HOMO and LUMO energy levels of these materials, while the molecular structures of all organic materials used in the device are listed in Fig. 5e. The obtained electroluminescence performances for green OLEDs are shown in Fig. 6, and the data are collected in Table 2. It can be seen from the current densityvoltage-luminance curves (Fig. 6a) that, the turn-on voltages and maximum luminance of the 
Control-G, H1-G and H2-G green devices are determined to be 3.0, 2.9 and $2.7 \mathrm{~V}, 115400$, 132800 and $108600 \mathrm{~cd} / \mathrm{m}^{2}$, respectively [24]. Furthermore, from the current efficiency-current density curves (Fig. 6b) and power efficiency-current density curves (Fig. 6c), we can learn that the maximum current efficiencies for Control-G, H1-G and H2-G green devices were 44.2, 51.7 and $37.8 \mathrm{~cd} / \mathrm{A}$, respectively, and the corresponding maximum power efficiency are $30.9,37.8$ and $34.5 \mathrm{~lm} / \mathrm{W}$, respectively. Fig. 6d exhibits the comparison of EQE of the Control-G, H1-G and H2-G green devices, showing the maximum EQE of 12.9, 15.0 and $13.0 \%$ for the three devices, respectively. The EL spectra of the above green devices at $6 \mathrm{~V}$ are shown in Fig. 6e. The maximum emission peak is found at $512 \mathrm{~nm}$ with the same Commission Internationale de L'Eclairage (CIE) coordinates of $(0.31,0.61)$. The EL spectra of device based on $\mathbf{H 1}$ at different applied voltages are shown in Fig. 6f. We can see that, the shape of the EL spectra does not show much change with the increase of the applied voltages which implies the high EL stability of device. Therefore, all the data as depicted above manifest that compounds $\mathbf{H 1}$ and $\mathbf{H 2}$ are excellent green host materials. Additionally, the green device based on host material H1 exhibits the best performance according to the aforementioned data. This could be attributed to the excellent thermal stability and high glass transition temperature of $\mathbf{H 1}$ and $\mathbf{H 2}$, which enhances the capability of forming a stable amorphous thin film. Besides, by comparing to $\mathbf{H} 2$ and $\mathbf{C B P}$, the higher LUMO and HOMO energy level of $\mathbf{H 1}$ can also block the hole and electron into the emitting layer, which could enhance the recombination chance of hole and electron and then benefits the improvement of device performance.

For blue OLEDs, the obtained electroluminescence performances are shown in Fig. 6 and the data are also collected in Table 2. It can be seen from the current density-voltage-luminance curves that (Fig. 7a), the turn-on voltages and maximum luminance of the Control-B, H1-B and H2-B blue devices are determined to be $3.3,3.2$ and $3.1 \mathrm{~V}, 15730,20360$ and $16640 \mathrm{~cd} / \mathrm{m}^{2}$, 
respectively. From the current efficiency-current density curves (Fig. 7b) and power efficiencycurrent density curves (Fig. 7c), it can be learnt that the maximum current efficiencies for Control-B, H1-B and H2-B blue devices were 14.3, 19.0 and $21.6 \mathrm{~cd} / \mathrm{A}$, respectively, and the corresponding maximum power efficiency are 9.8, 14.7 and $17.2 \mathrm{~lm} / \mathrm{W}$, respectively. Fig. $7 \mathrm{~d}$ exhibits the comparison of EQE of the Control-B, H1-B and H2-B blue devices, showing the maximum EQE of $6.4,8.5$ and $8.8 \%$ for the three devices, respectively. The EL spectra comparison of the above blue devices at $6 \mathrm{~V}$ are shown in Fig. 7e. The maximum emission peak is found at around $476 \mathrm{~nm}$ with the Commission Internationale de L'Eclairage (CIE) coordinates of $(0.18,0.28),(0.18,0.37)$ and $(0.21,0.41)$ for Control-B, H1-B and H2-B, respectively. The EL spectra of device based on $\mathbf{H 2}$ at different applied voltages are displayed in Fig. 7f. We can observe clearly that, the shape of the EL spectra does not show much change with the increase of the applied voltages which implies the high EL stability of device H2-B. Therefore, all the data as depicted above manifest that compounds $\mathbf{H 1}$ and $\mathbf{H 2}$ are proven to be also excellent blue host materials. Additionally, blue PHOLED device based on H2 exhibits the best performance according to the above data. This might be ascribed to the higher triplet energy level of $\mathbf{H 2}$, which facilitate the effective energy transfer from host to emitter and then benefits the improvement of device performance [29,36].

As discussed above, the bipolar host materials $\mathbf{H 1}$ and $\mathbf{H 2}$ exhibit higher current efficiency and power efficiency for both green and blue PHOLEDs as compared to the commercial host materials $\mathrm{CBP}$ and $\mathrm{mCP}$. Therefore, combining the triazole and carbazole derivatives is a good method to enhance the performance of host materials in OLEDs, which also enlarges the category of organic functional groups to serve as host materials. It should be pointed out that the electroluminescent performance was obtained under ordinary laboratory condition where the 
device is not packaged. The device performance could be further improved by optimization of the processing conditions [37].

\subsection{Morphology study}

To further understand the mechanism underlying electroluminescent efficiency enhancement of green and blue OLEDs with $\mathbf{H 1}$ and $\mathbf{H 2}$ as the host, we then performed atomic force microscopy (AFM) measurements to investigate the morphology of thin films of $\mathbf{H 1}$ and $\mathbf{H 2}$ by comparing with the thin films of commercial host materials CBP and mCP. As shown in Fig. 8, it can be seen that, the surface roughness of $\mathbf{H 1}$ and $\mathbf{H 2}$ are lower than those of the commercial host materials, especially that of CBP. Moreover, the surface roughness of $\mathbf{H 2}$ is much smaller than that of mCP, which can also explain its outstanding performance in blue PHOLED. Hence, the morphology study reveals a crucial influence factor responsible for the improvement of PHOLEDs with $\mathbf{H 1}$ and $\mathbf{H} 2$ as the host.

\section{Conclusions}

To conclude, two novel bipolar host materials $\mathbf{H 1}$ and $\mathbf{H 2}$ based on triazole/carbazole derivatives were designed and synthesized. Their thermal, photophysical, electrochemical and electroluminescent properties were fully investigated. Both H1 and $\mathbf{H 2}$ exhibit good thermal properties with the decomposition temperatures $\left(\mathrm{T}_{\mathrm{d}}\right)$ close to $400{ }^{\circ} \mathrm{C}$. Due to their high thermal stability, favorable photophysical, electrochemical and bipolar charge-transport properties, excellent electroluminescent performances for both green and blue PHOLED were obtained with $\mathrm{H1}$ and $\mathrm{H2}$ as the host materials. H1 exhibits the best device performances with the maximum current efficiency, maximum power efficiency and maximum brightness of $51.7 \mathrm{~cd} / \mathrm{A}, 37.8 \mathrm{~lm} / \mathrm{W}$ and $132800 \mathrm{~cd} / \mathrm{m}^{2}$, respectively, for green PHOLEDs, while $\mathbf{H 2}$ shows the best device performances with the maximum current efficiency, maximum power efficiency and maximum brightness of $21.6 \mathrm{~cd} / \mathrm{A}, 17.2 \mathrm{~lm} / \mathrm{W}$ and $16640 \mathrm{~cd} / \mathrm{m}^{2}$, respectively, for blue PHOLED due to its 
higher triplet energy level. It was of important scientific significance to discover novel host materials for high efficiency OLEDs by combining the triazole and carbazole derivatives. Further investigation along this direction is in progress.

\section{Acknowledgements}

We acknowledge the financial support from the National Natural Science Foundation of China (Grant No.: 61307030, 21303117). This work was also supported by the Program for the Outstanding Innovative Teams of Higher Learning Institutions of Shanxi (OIT), Fund Program for the Scientific Activities of Selected Returned Overseas Professionals in Shanxi Province, the Natural Science Foundation for Young Scientists of Shanxi Province, China (Grant No.: 2014021019-2), the Outstanding Young Scholars Cultivating Program, Research Project Supported by Shanxi Scholarship Council of China (Grant No.: 2014-02) and the Key R\&D Project of Shanxi Province (International cooperation program, No. 201603D421032). W. Y. Wong thanks for the financial support from the Hong Kong Polytechnic University.

\section{References}

1 Baldo MA, O'Brien DF, You YJ, et al. High efficiency phosphorescent emission from organic electroluminescent devices. Nature 1998; 395: 151-154.

2 Tao P, Li WL, Zhang J, et al. Adv. Funct. Mater. 2016; 26: 881-894.

3 Uoyama H, Goushi K, Shizu K, et al. Highly efficient organic light-emitting diodes from delayed fluorescence. Nature 2012; 492: 234-237.

4 Yang XL, Zhou GJ, Wong WY. Functionalization of phosphorescent emitters and their host materials by main group elements for phosphorescent organic light - emitting devices. Chem. Soc. Rev. 2015; 44: 8484-8575.

5 Adachi C, Baldo MA, Thompson ME, et al. Nearly 100\% internal phosphorescence efficiency in an 
organic light-emitting device. J. Appl. Phys. 2001; 90: 5048-5051.

6 Sun H, Liu S., Lin W, et al. Smart responsive phosphorescent materials for data recording and security protection. Nat. Commun. 2016; 28: 7137-7142.

7 Zhang KY, Chen X, Sun G, et al. Adv. Mater. 2011; 40: 2943-2970.

8 Zhao Q, Xu W, Sun H, et al. Tunable electrochromic luminescence of iridium(III) complexes for information self-encryption and anti-counterfeiting. Adv. Optical Mater. 2016, 4: 1167-1173.

9 Cui LS, Xie YM, Wang YK, Zhong C, Deng XY, Liu XY. Pure hydrocarbon hosts for $\approx 100 \%$ exciton harvesting in both phosphorescent and fluorescent light - emitting devices. Adv. Mater. 2015; 27: 4213-4217.

10 Lin W, Zhao Q, Sun H, et al. An electrochromic phosphorescent iridium(III) complex for information recording, encryption, and decryption. Adv. Optical Mater. 2015, 3: 368-375.

11 Kim SY, Jeong WI, Mayr C, Park YS, Kim KH. Light - emitting diodes: organic light - emitting diodes with $30 \%$ external quantum efficiency based on a horizontally oriented Emitter . Adv. Funct. Mater. 2013; 23: 3896-3900.

12 Zhuo MJ, Sun W, Liu GW, Wang J, Guo LY, Liu C. Pure aromatic hydrocarbons with rigid and bulky substituents as bipolar hosts for blue phosphorescent OLEDs. J. Mater. Chem. C 2015; 3: 9137-9144.

13 Yook KS, Lee JY, et al. Organic materials for deep blue phosphorescent organic light-emitting diodes. Adv. Mater. 2012; 24: 3169-3172.

$14 \mathrm{Su} \mathrm{SJ}$, Sasabe H, Pu YJ, et al. Tuning energy levels of electron-transport materials by nitrogen orientation for electrophosphorescent devices with an 'ideal' operating voltage. Adv. Mater. 2010; 22: 3311.

15 Tao YT, Wang Q, Yang CL, Zhang ZQ. A simple carbazole/oxadiazole hybrid molecule: an excellent 
bipolar host for green and red phosphorescent OLEDs. Angew. Chem. Int. Ed. 2008; 47: 8104-8107.

16 Holder E, Langeveld BMW, Schubert U. New trends in the use of transition metal-Ligand complexes for applications in electroluminescent devices. Adv. Mater 2005; 17: 1109-1121.

17 Huang JH, Su JH, Tian H. The development of anthracene derivatives for organic light-emitting diodes. J. Mater. Chem. 2012; 22: 10977-10989.

18 Tian GJ, Wei X, Xiang N, Huang JH, Cao J, Wang ZX. Small organic molecules based on oxazole/thiazole with excellent performances in green and red phosphorescent organic light-emitting diodes. RSC Adv. 2016; 6: 51575-51582.

19 Kim HS, Kim YJ, Lee HK, et al. Activation of macrophages by polysaccharide isolated from Paecilomyces cicadae through toll-like receptor. Chem. -Asian J. 2012; 50: 3190-7.

20 Kim GW, Yang DR, Yong CK, Fan, CH. Chai KY,. Kwon JH. Di(biphenyl)silane and carbazole based bipolar host materials for highly efficient blue phosphorescent OLEDs. Dyes and Pigments 2016; 136: $8-16$.

21 Hu MM, Liu Y, Chen Y, Song WX, Gao L, Mu HC, et al. Highly efficient triazine/carbazole-based host material for green phosphorescent organic light-emitting diodes with low efficiency roll-off. RSC Adv. 2017; 7: 7287-7292.

22 Cui LS, Dong SC, Liu Y, Xu MF, Li Q, Jiang ZQ. Meta -Linked spirobifluorene/phosphine oxide hybrids as host materials for deep blue phosphorescent organic light-emitting diodes. Organic Electronics 2013; 14: 1924-1930.

23 Yang XL, Xu XB, Zhou GJ. Recent advances of the emitters for high performance deep-blue organic light-emitting diodes. J. Mater. Chem. C 2014; 3: 913-944.

24 Promarak V, Saengsuwan S, Jungsuttiwong S, Sudyoadsuk T, Keawin T, et al. Synthesis and 
characterization of N -carbazole end-capped oligofluorenes. Tetrahedron Lett. 2007; 48: 89-93.

25 Konidena RK, Thomas KRJ, Sahoo S, Dubey DK, Jou JH. Multi-substituted deep-blue emitting carbazoles: a comparative study on photophysical and electroluminescence characteristics. J. Mater. Chem. C 2016; 5: 709-726.

26 Bai Q, Liu H, Yao L, Shan T, Gao Z, Zhang Y, Li P, Lu B. Adjusting nitrogen atom orientations of pyridine ring in tetraphenylsilane-based hosts for highly efficient blue phosphorescent OLEDs. ACS Appl. Mater. Interfaces 2016; 8: 4793-248.

27 Kim MK, Kwon J, Kwon TH, Hong JI, et al. A bipolar host containing 1,2,3-triazole for realizing highly efficient phosphorescent organic light-emitting diodes. New J. Chem. 2010; 34: 1317-1322.

28 Wagner D, Hoffmann ST, Heinemeyer U, Münster I, Köhler A, Strohriegl P. Triazine based bipolar host materials for blue phosphorescent OLEDs. Chem. Mater. 2013; 25: 3758-3765.

29 Kim M, Jeon SK, Hwang SH, Lee JY. Molecular design of triazine and carbazole based host materials for blue phosphorescent organic emitting diodes. Phys. Chem. Chem. Phys. 2015; 17: 13553-13558.

30 Zheng Z, Dong Q, Gou L, Su JH, Huang J. Novel hole transport materials based on N,Nprimedisubstituted-dihydrophenazine derivatives for electroluminescent diodes. J. Mater. Chem. C 2014; 2: 9858-9865.

31 Huang JH, Su JH, Li X, Lam MK, Fuang KM, Fan HH. Bipolar anthracene derivatives containing hole- and electron-transporting moieties for highly efficient blue electroluminescence devices. J. Mater. Chem. 2011; 21: 2957-2964.

32 Jeon SO, Lee JY, et al. Fluorenobenzofuran as the core structure of high triplet energy host materials for green phosphorescent organic light-emitting diodes. J. Mater. Chem. 2012; 22: 10537-10541.

33 Chen DC, Liu KK, Gan L, Liu M, Gao K, Xie GZ. Modulation of exciton generation in organic active 
planar pn heterojunction: Toward low driving voltage and high-efficiency OLEDs employing conventional and thermally activated delayed fluorescent emitters. Adv. Mater. 2016; 28: 6758-6765.

34 Rothmann MM, Fuchs E, Schildknecht C, Schidknecht, N. Langer, C. Lennartz, I. Munster, P. Designing a bipolar host material for blue phosphorescent OLEDs: Phenoxy-carbazole substituted triazine. Organic Electronics 2011; 12: 1192-1197.

35 W Li W, Li J, Liu D, Jin Q, et al. Simple Bipolar Host materials for high-efficiency blue, green and white phosphorescence OLEDs. ACS Appl. Mater. Interfaces 2016; 8: 22382-22391.

36 Rothmann MM, Haneder S, Como ED, Lennartz C, Schidknecht C, Strohriegl P. Donor-Substituted 1,3,5-Triazines as Host materials for blue phosphorescent organic light-emitting diodes. Chem. Mater. 2010; 22: 2403-2410.

37 Y. Chen, J. Xie, Z. Wang, J. Cao, H. Chen, J. Huang, Dyes and Pigments, 2016, 124, 188-195. Chen Y, Xie J, Wang Z, Cao J, Chen H, Huang J. Highly efficient bipolar host material based-on indole and triazine moiety for red phosphorescent light-emitting diodes. Dyes and Pigments 2016; 124: 188-195. 
Table 1 The physical properties of compound $\mathbf{H 1}$ and $\mathbf{H 2}$.

\begin{tabular}{ccccccccccc}
\hline $\begin{array}{c}\text { Compou } \\
\text { ndl }\end{array}$ & $\begin{array}{c}\lambda_{\max } \\
\mathrm{abs}^{\mathrm{a}} \\
(\mathrm{nm})\end{array}$ & $\begin{array}{c}\lambda_{\max } \mathrm{em}^{\mathrm{a}} \\
(\mathrm{nm})\end{array}$ & $\begin{array}{c}\mathrm{E}_{\mathrm{g}} \mathrm{b} \\
(\mathrm{eV})\end{array}$ & $\begin{array}{c}\mathrm{HOMO}^{\mathrm{c}} \\
(\mathrm{eV})\end{array}$ & $\begin{array}{c}\mathrm{LUMO}^{\mathrm{d}} \\
(\mathrm{eV})\end{array}$ & $\begin{array}{c}\mathrm{E}_{\mathrm{T}}{ }^{\mathrm{e}} \\
(\mathrm{eV})\end{array}$ & $\begin{array}{c}\mathrm{T}_{\mathrm{g}} \mathrm{f}^{\mathrm{g}} \\
\left({ }^{\circ} \mathrm{C}\right)\end{array}$ & $\begin{array}{c}\mathrm{T}_{\mathrm{d}^{\mathrm{g}}} \\
\left({ }^{\circ} \mathrm{C}\right)\end{array}$ & $\begin{array}{c}\Phi^{\mathrm{h}} \\
(\%)\end{array}$ & $\begin{array}{c}\tau^{\mathrm{i}} \\
(\mathrm{ns})\end{array}$ \\
\hline $\mathrm{H} 1$ & 279 & 416 & 3.22 & -6.09 & -2.87 & 2.64 & 94.74 & 383.93 & 9 & 2.84 \\
\hline $\mathrm{H} 2$ & 273 & 416 & 3.29 & -6.09 & -2.70 & 2.70 & 100.05 & 386.47 & 16 & 2.42 \\
\hline
\end{tabular}

${ }^{\mathrm{a}}$ Measured in THF; ${ }^{\mathrm{b}}$ Estimated from onset of the absorption spectra $\left(\mathrm{E}_{\mathrm{g}}=1240 / \lambda_{\text {onset }}\right) ;{ }^{\mathrm{c}}$ Calculated from cyclic voltammetry; ${ }^{\mathrm{d} C a l c u l a t e d}$ by the equation $\mathrm{E}_{\mathrm{HOMO}}=\mathrm{E}_{\mathrm{LUMO}}-\mathrm{E}_{\mathrm{g}}$; ${ }^{\mathrm{e}}$ Calculated by the first peak of phosphorescent spectra measured at $77 \mathrm{~K}$; ${ }^{\mathrm{f}}$ Measured by DSC; ${ }^{\mathrm{g}}$ Measured by TGA. ${ }^{\mathrm{h}}$ Fluorescence quantum yield are measured in $\mathrm{CH}_{2} \mathrm{Cl}_{2}$ relative to quinine sulphate; ${ }^{\mathrm{g}}$ Measured at room temperature in 2-methyltetrahydrofuran solution with the excitation wavelength at $375 \mathrm{~nm}$.

Table 2 A summary of parameters measured for a set of structurally identical green and blue PHOLEDs with different host materials, the performance of the relative control devices are also listed for comparison.

\begin{tabular}{ccccccc}
\hline Device & $\begin{array}{c}\mathrm{V}_{\mathrm{on}}{ }^{\mathrm{a}} \\
(\mathrm{V})\end{array}$ & $\begin{array}{c}\lambda_{\mathrm{EL}} \\
(\mathrm{nm})\end{array}$ & $\begin{array}{c}\mathrm{CE}^{\mathrm{b}} \\
(\mathrm{cd} / \mathrm{A})\end{array}$ & $\begin{array}{c}\mathrm{PE}^{\mathrm{c}} \\
(\mathrm{m} / \mathrm{W})\end{array}$ & $\begin{array}{c}\text { Max. } \\
\text { Luminance } \\
\left(\mathrm{cd} / \mathrm{m}^{2}\right)\end{array}$ & $\begin{array}{c}\mathrm{EQE}^{\mathrm{d}} \\
(\%)\end{array}$ \\
\hline Control-G & 3.0 & 512 & 44.2 & 30.9 & 115400 & 12.9 \\
\hline H1-G & 2.9 & 512 & 51.7 & 37.8 & 132800 & 15.0 \\
\hline H2-G & 2.7 & 512 & 45.5 & 34.5 & 108600 & 13.0 \\
\hline Control-B & 3.3 & 472 & 14.3 & 9.80 & 15730 & 6.4 \\
\hline H1-B & 3.2 & 476 & 19.0 & 14.7 & 20360 & 8.5 \\
\hline H2-B & 3.1 & 476 & 21.6 & 17.2 & 16640 & 8.8 \\
\hline
\end{tabular}

${ }^{a}$ Turn-on voltage, recorded at luminance of $1 \mathrm{~cd} \mathrm{~m}^{-2}$; ${ }^{\mathrm{b}}$ Maximum current efficiency; ${ }^{\mathrm{c}}$ Maximum power efficiency; ${ }^{\mathrm{d}}$ External quantum efficiency. 
Fig. 1 (A) DSC and (B) TGA curves of $\mathbf{H 1}$ and $\mathbf{H 2}$.

Fig. 2 Absorption spectra, photoluminescence spectra (at room temperature in THF) and phosphorescence spectra (at $77 \mathrm{~K}$ in THF) of $\mathbf{H 1}$ and $\mathbf{H 2}$ at $1.0 \times 10^{-5} \mathrm{~mol} / \mathrm{L}$.

Fig. 3 Cyclic voltammograms of $\mathbf{H 1}$ and $\mathbf{H 2}$ in toluene with tetra $(n$ butyl)ammoniumhexafluorophosphate $(0.1 \mathrm{M})$ as the supporting electrolyte for the oxidation scan. Fig. 4 Current density-voltage curves of the (A) hole-only and (B) electron-only devices for CBP, $\mathrm{mCP}, \mathbf{H 1}$ and $\mathbf{H 2}$.

Fig. 5 (A) Schematic drawing illustrating the device structure and (B) energy level diagram of green PHOLEDS; (C) Schematic drawing illustrating the device structure and (D) energy level diagram of blue PHOLEDS; (E) molecular structures of organic materials as-used in the PHOLEDs.

Fig. 6 (A) Current density-voltage-luminance curves, (B) current efficiency-current density curves, (C) power efficiency-current density curves and (D) EQE-brightness characteristics measured for Control-G, H1-G and H2-G, (E) electroluminescence spectra of green PHOLEDs at $6 \mathrm{~V}$ and $(\mathbf{F})$ electroluminescence spectra of green PHOLED based on $\mathbf{H 1}$ at different applied voltages.

Fig. 7 (A) Current density-voltage-luminance curves, (B) current efficiency-current density curves, (C) power efficiency-current density curves and (D) EQE-brightness characteristics measured for Control-B, H1-B and H2-B, (E) electroluminescence spectra of blue PHOLEDs at $6 \mathrm{~V}$ and (F) electroluminescence spectra of blue PHOLED based on $\mathbf{H 2}$ at different applied voltages.

Fig. 8 AFM images of the thermally evaporated thin films of (a) CBP, (b) $\mathbf{m C P}$, (c) H1 and (d) H2, respectively.

Scheme 1 Synthetic routes of bipolar host materials $\mathbf{H 1}$ and $\mathbf{H 2}$. 

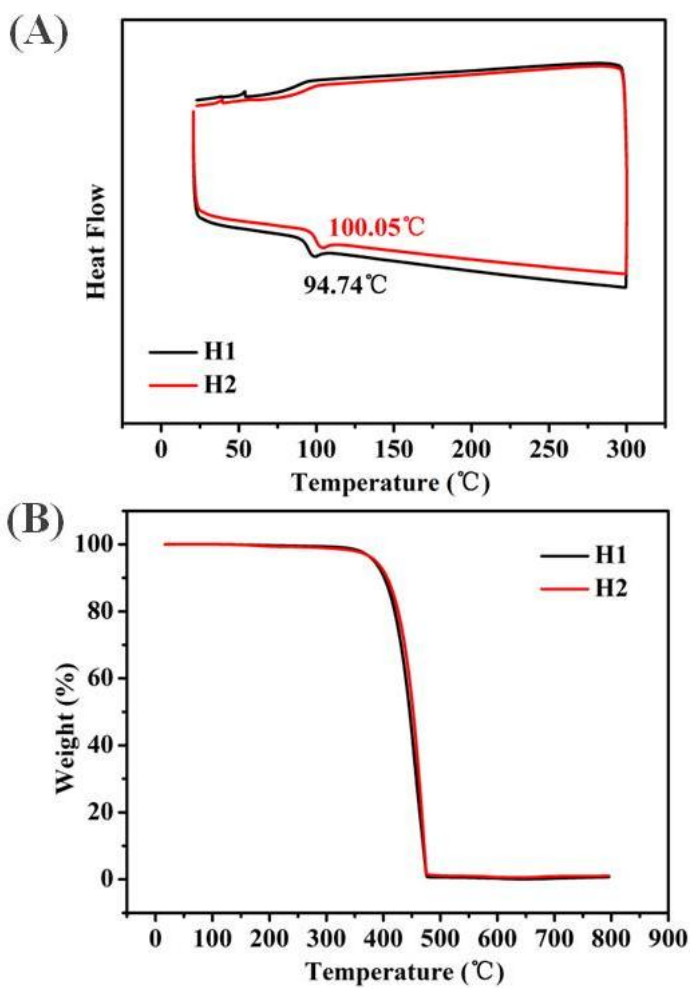

Fig. 1 (A) DSC and (B) TGA curves of $\mathbf{H 1}$ and $\mathbf{H 2}$.

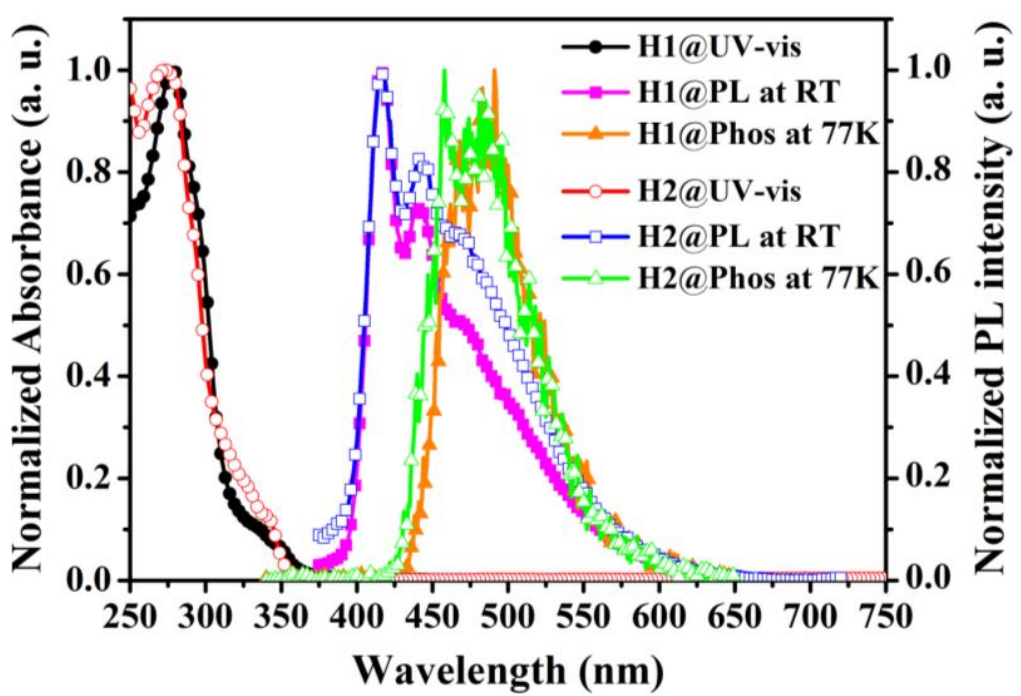

Fig. 2 Absorption spectra, photoluminescence spectra (at room temperature in THF) and phosphorescence spectra (at $77 \mathrm{~K}$ in THF) of $\mathbf{H 1}$ and $\mathbf{H 2}$ at $1.0 \times 10^{-5} \mathrm{~mol} / \mathrm{L}$. 


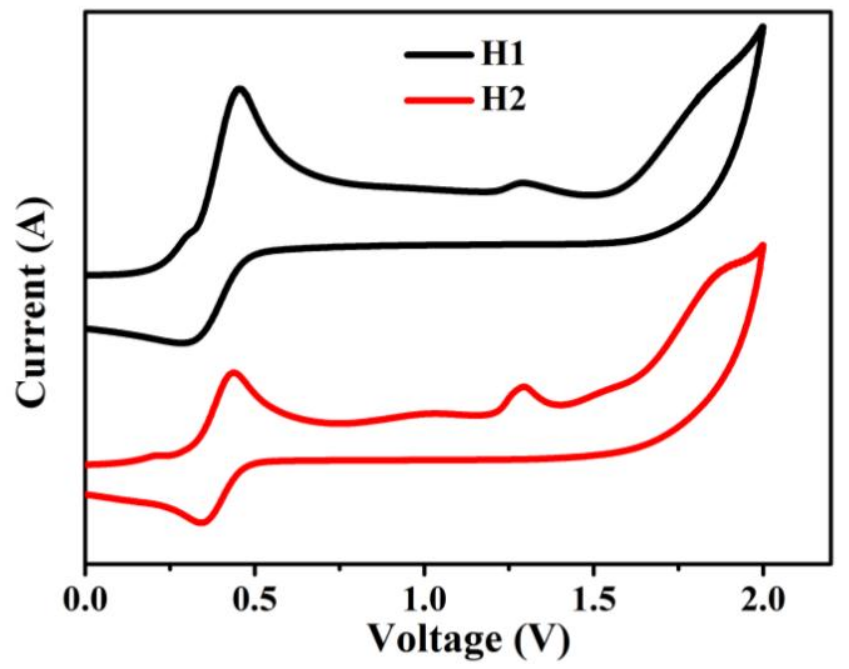

Fig. 3 Cyclic voltammograms of $\mathbf{H 1}$ and $\mathbf{H 2}$ in toluene with tetra $(n$ butyl)ammoniumhexafluorophosphate $(0.1 \mathrm{M})$ as the supporting electrolyte for the oxidation scan.
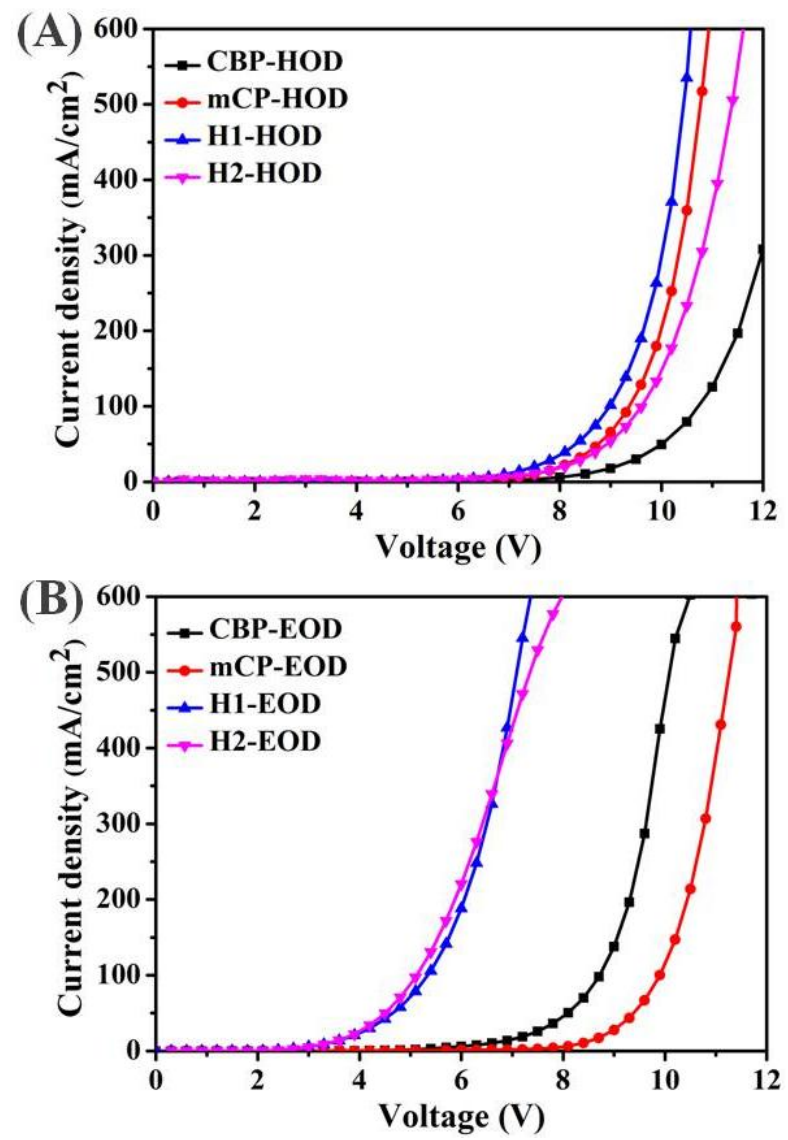

Fig. 4 Current density-voltage curves of the (A) hole-only and (B) electron-only devices for CBP, $\mathrm{mCP}, \mathbf{H 1}$ and $\mathbf{H 2}$. 

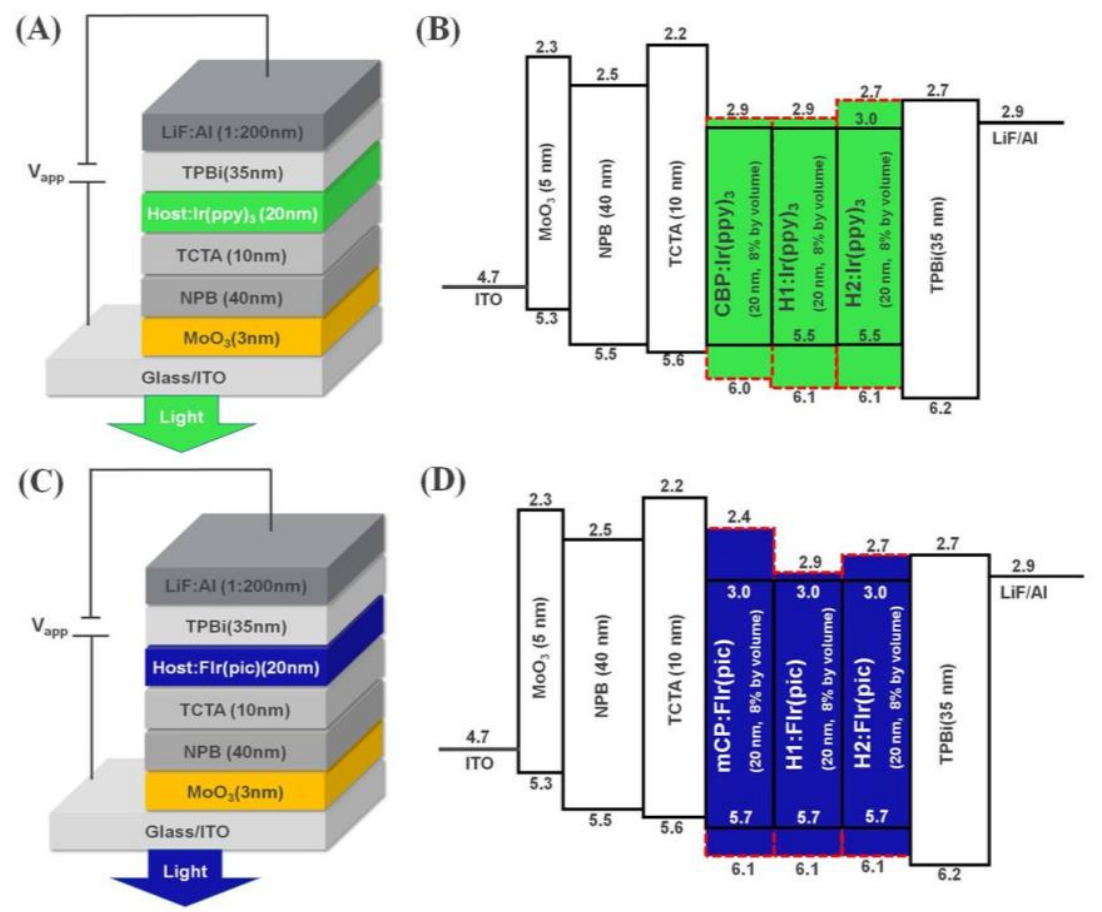

(E)
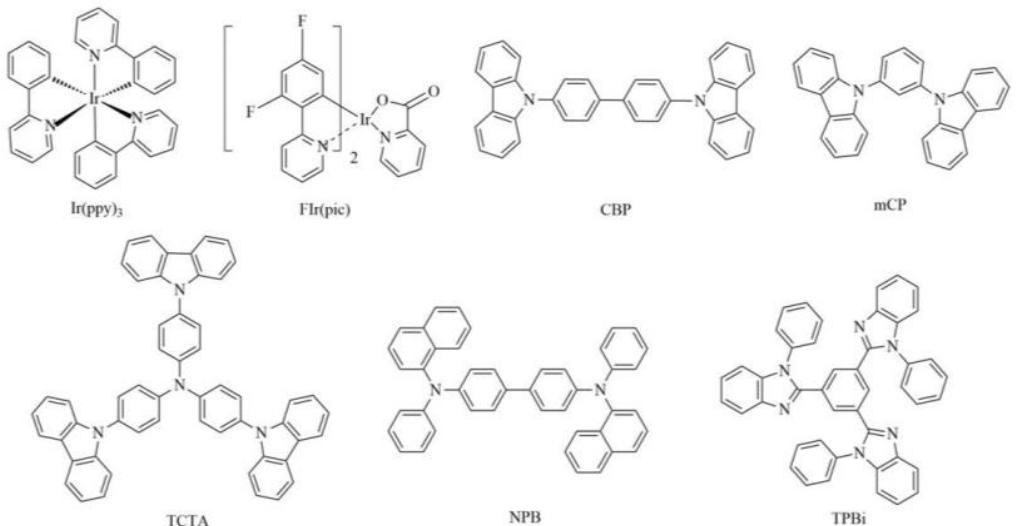

Fig. 5 (A) Schematic drawing illustrating the device structure and (B) energy level diagram of green PHOLEDS; (C) Schematic drawing illustrating the device structure and (D) energy level diagram of blue PHOLEDS; (E) molecular structures of organic materials as-used in the PHOLEDs. 

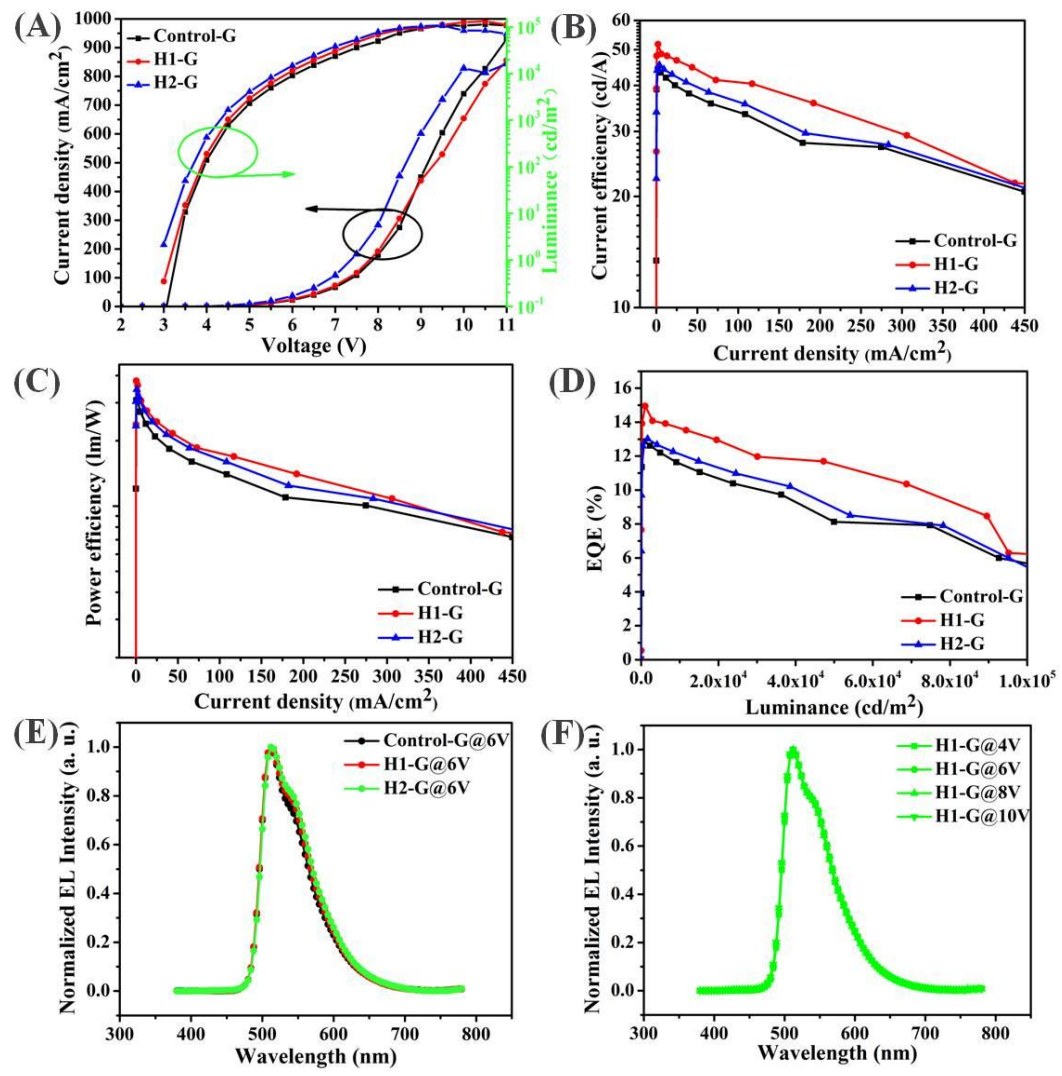

Fig. 6 (A) Current density-voltage-luminance curves, (B) current efficiency-current density curves, (C) power efficiency-current density curves and (D) EQE-brightness characteristics measured for Control-G, H1-G and H2-G, (E) electroluminescence spectra of green PHOLEDs at $6 \mathrm{~V}$ and $(\mathbf{F})$ electroluminescence spectra of green PHOLED based on $\mathbf{H 1}$ at different applied voltages. 

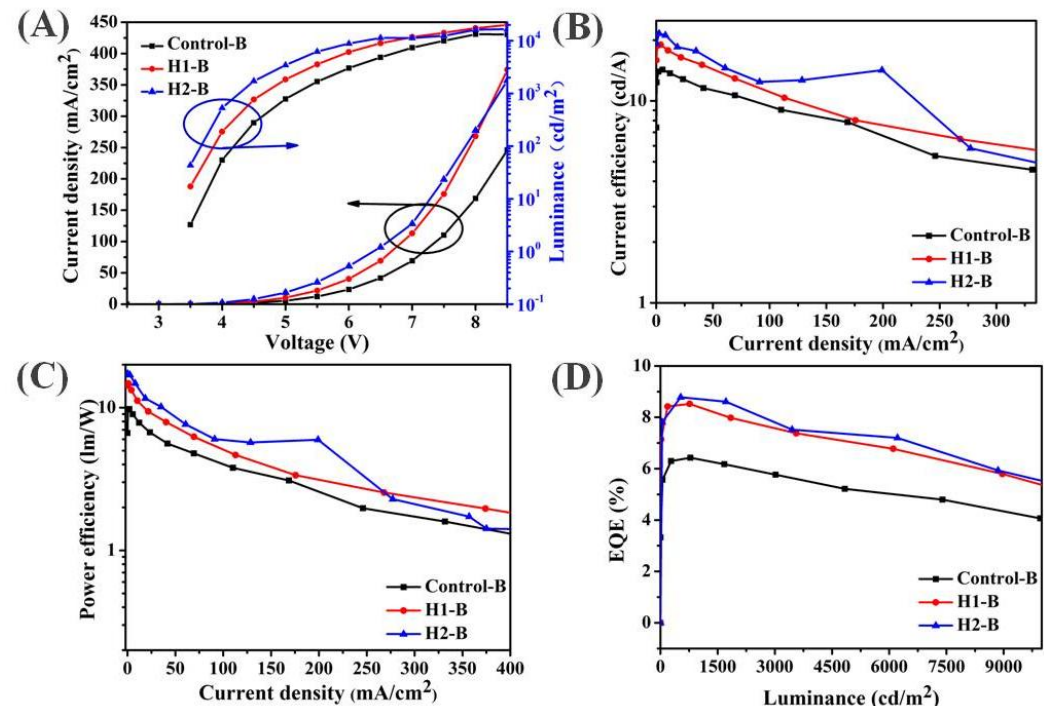

(D)
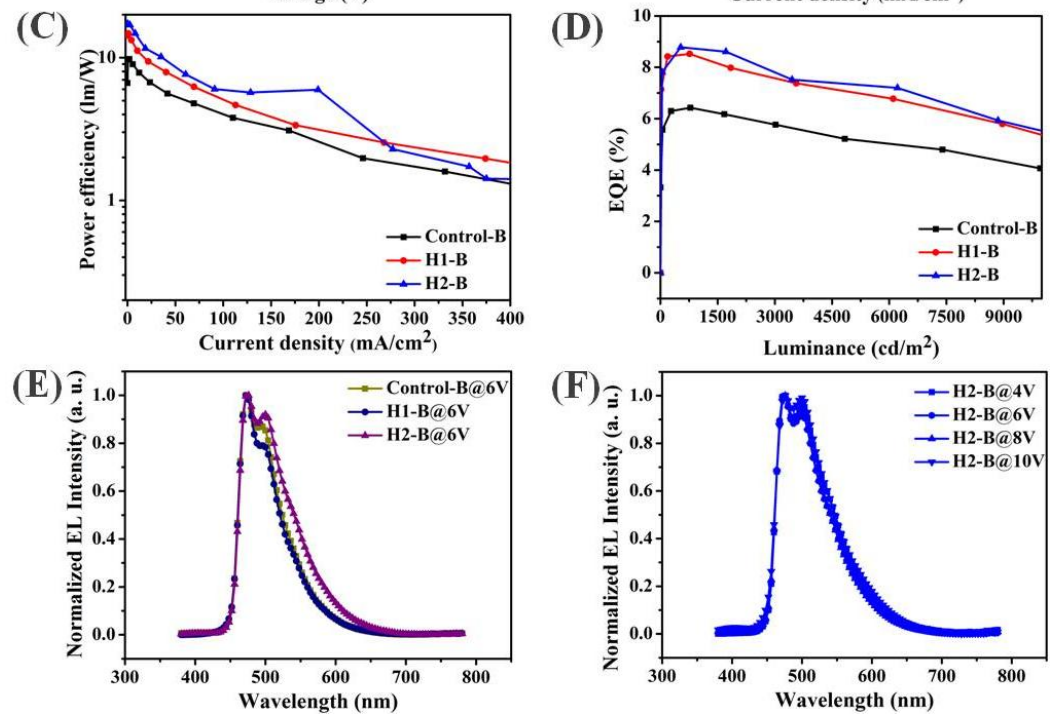

Fig. 7 (A) Current density-voltage-luminance curves, (B) current efficiency-current density curves, (C) power efficiency-current density curves and (D) EQE-brightness characteristics measured for Control-B, H1-B and H2-B, (E) electroluminescence spectra of blue PHOLEDs at $6 \mathrm{~V}$ and (F) electroluminescence spectra of blue PHOLED based on $\mathbf{H 2}$ at different applied voltages. 

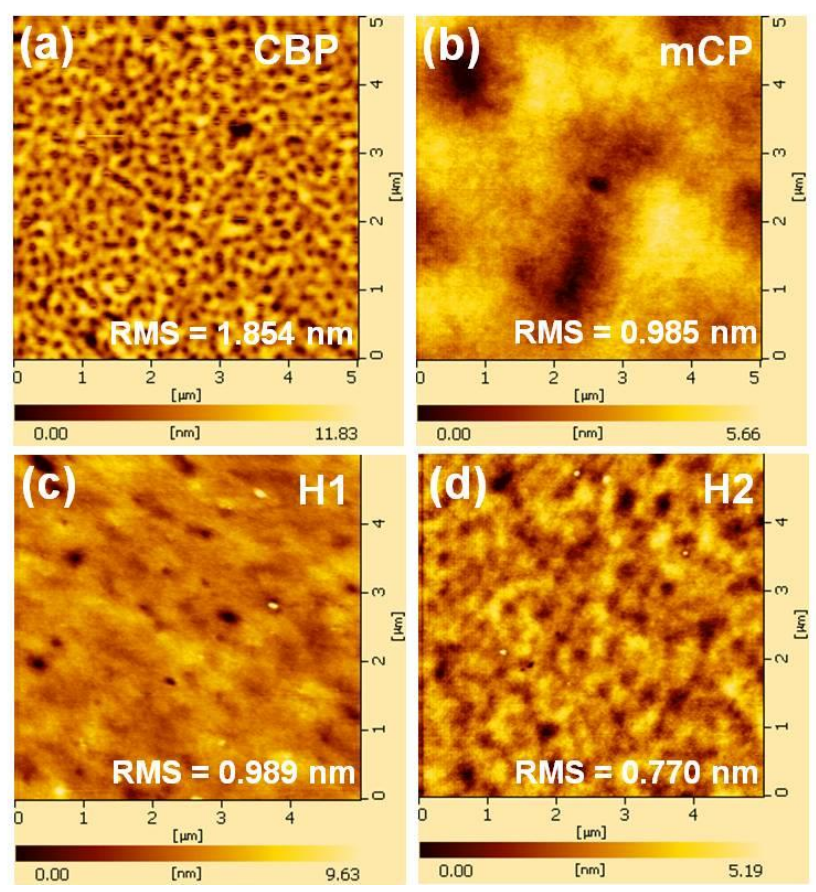

Fig. 8 AFM images of the thermally evaporated thin films of (a) CBP, (b) $\mathbf{m C P}$, (c) H1 and (d) H2, respectively.

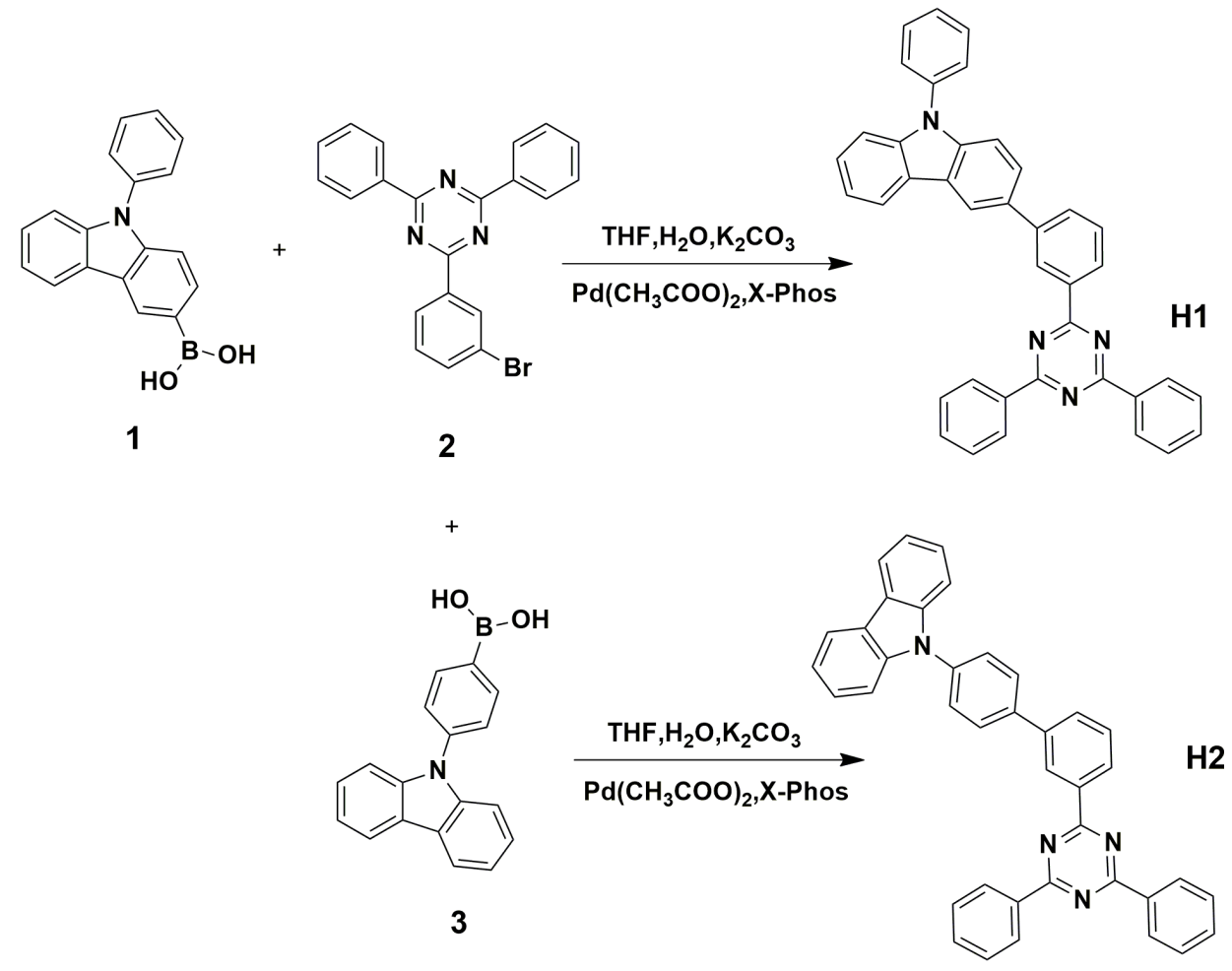

Scheme 1 Synthetic routes of bipolar host materials $\mathbf{H 1}$ and $\mathbf{H 2}$. 